\title{
UTILIZAÇÃO DA MORFOLOGIA FUZZY PARA QUANTIFICAÇÃO DE ESPOROS DE FUNGOS MICORRIZICOS
}

\author{
Alécio Santos Barros \\ Dept. de Ciências Exatas e \\ Tecnológicas \\ UESB \\ Vitoria da Conquista, Bahia, Brasil. \\ aleciochaos@gmail.com
}

\author{
Alexsandra Oliveira Andrade \\ Dept. de Ciências Exatas e \\ Tecnológicas \\ UESB \\ Vitoria da Conquista, Bahia, Brasil \\ alexsandra@uesb.edu.br
}

\author{
Roque Mendes P. Trindade \\ Dept. de Ciências Exatas e \\ Tecnológicas \\ UESB \\ Vitoria da Conquista, Bahia, Brasil \\ roque@uesb.edu.br
}

\begin{abstract}
Resumo - Este trabalho apresenta um método de contagem de esporos de fungos micorrízicos a partir de imagens digitais usando morfologia fuzzy para separação de esporos e algoritmos desenvolvidos no Scilab para quantificação. O objetivo é mostrar a eficácia da morfologia fuzzy e as funções desenvolvidas na separação e contagem. A estratégia metodológica desenvolvida foi utilizar as definições de morfologia fuzzy desenvolvidas por Andrade et al e produzir um algoritmo capaz de separar e contar fungos. Espera-se obter um método capaz de realizar uma contagem eficiente.
\end{abstract}

Palavras Chaves - morfologia fuzzy, contagem, esporos, Scilab, processamento de imagem.

\section{INTRODUÇÃO}

A área de processamento de imagens digital tem um papel importante na vida humana. Atualmente, o campo de processamento de imagens tem inúmeras aplicações comerciais, científicas, industriais, médicas, militares, de entretenimento e outras. As pesquisas científicas, e o desenvolvimento de novas tecnologias de alto padrão resultaram em tais aplicações. Essa interação continua mantendo esta área de pesquisa ativa e bastante ampla. Alguns tópicos bem conhecidos em processamento de imagens digitais como melhoria da qualidade da imagem (filtragem, redução de ruídos, realce, restauração), analise de imagem (detecção de borda, segmentação, reconhecimento e contagem de objetos, interpretação), compressão de imagem e reconstrução de imagem.

A fim de lidar com todos esses e outros problemas, várias técnicas foram desenvolvidas e introduzidas, muitas vezes com grande sucesso. Dentre as diferentes técnicas que estão atualmente em uso, encontram-se as técnicas fuzzy. Bouchet et al (2007) propuseram um método para segmentação de imagens de angiografia retinal usando morfologia fuzzy para melhorar o diagnóstico de profissionais da saúde. Boaventura (2010) propõe uma técnica para processamento de imagem e detecção de borda utilizando números fuzzy e morfologia matemática fuzzy. Estes são alguns exemplos do uso de tal técnica. Baseado em muitas experiências bem sucedidas em aplicações da teoria de conjunto fuzzy, as técnicas fuzzy agregam efetivamente valores as áreas de processamento de imagem e visão computacional.

Uma das principais aplicações do processamento digital de imagens é a contagem de certos objetos em uma imagem. O termo "objeto" refere-se aqui a qualquer elemento de interesse que pode ser identificado numa imagem digital. A variedade de problemas de contagem de objetos é muito grande, cada qual com suas características e desafios intrínsecos. O grau de dificuldade do problema depende de uma série de fatores: contraste entre os objetos e o fundo, grau de agrupamento dos objetos, textura dos objetos e sua variação, tamanho do objeto e sua variação, complexidade dos objetos, etc.

Várias estratégias foram propostas para solucionar uma grande variedade de problemas de contagem de objetos como células, bactérias, árvores, frutas, amostra de solo, fungos, pólen, espigas, entre outras (BARBEDO 2012). Salis \& Pereira (2006) propuseram um método para a contagem automática de tarugos de aço na linha de produção de siderúrgicas, obtendo bons resultados utilizando ferramentas como filtragens para a remoção de ruído e correção de brilho, limiarização, erosão e dilatação. Poomcokrak \& Neatpisarnvanit (2008) desenvolveram um método simples de contagem de células vermelhas do sangue que consiste em eliminar as células incompletas, extrair as células individuais com detecção de borda usando morfologia matemática e identificação e contagem usando redes neurais. Men et al (2008) apresentaram um método para contagem de bactérias heterotróficas em água de refrigeração industrial que utiliza a conversão para tons de cinza e binarização usando o método de limiar com ponderação pela escala de cinza. Os trabalhos citados são alguns dentre muitos que se utilizam de técnicas de processamento de imagem para contagem de objetos.

Este trabalho tem como objetivo mostrar a eficiência dos operadores morfológicos fuzzy propostos por ANDRADE (2014), na separação de objetos, para isso serão realizados testes buscando identificar o melhor operador e o melhor elemento. Um algoritmo de contagem será utilizado como meio de medir esta eficiência.

\section{PROBLEMA}

A maioria dos organismos vivos estabelecem associações com outros organismos com a finalidade de garantir a sobrevivência e entre estas estão a associação micorrizas, do grego mico [fungo] e riza [raiz].

Vários são os tipos de associações micorrízicas, sendo algumas delas muito específicas, encontradas em apenas algumas famílias de plantas terrestres (BERBARA, 1996), no entanto levantamentos indicam que $80 \%$ das famílias de plantas são formadas por espécies que formam micorrizas arbusculares (doravante MA) que são encontradas em todas as latitudes e em quase todos os 
ecossistemas terrestres (BERBARA, 1996). Os fungos MAs são classificados como pertencentes ao filo Glomeromycota, classe Glomeromycetes (REDECKER, 2013).

Os esporos mais especificamente chamados de glomerosporos (GOTO, 2006) germinam e suas hifas se desenvolvem junto ao sistema radicular das plantas.

Um dos procedimentos metodológicos básicos ao estudo das micorrizas arbusculares é a extração dos glomerosporos do solo (peneiramento úmido). O método do peneiramento úmido consiste em pegar porções de terra com massa conhecida, misturá-las em água, sendo a mistura passadas através de peneiras e centrifugadas, repetidas vezes. Posteriormente uma solução de sacarose é acrescentado à mistura para as últimas centrifugações. Ao final deste procedimento, será obtida uma determinada quantidade de glomerosporos que necessitará ser quantificada. Essa contagem é realizada manualmente. Os glomerosporos são transferidos para uma placa de Petri e para auxiliar na visualização dessas estruturas que variam de 22 a $1050 \mu \mathrm{m}$ (SOUZA, 2010) um microscópio estereoscópico (lupa) é utilizado. Outros tipos de auxílios também são utilizados como o uso de uma folha quadriculada sob a placa de Petri para servir de orientação ou placa de Petri com círculos concêntricos (placa canelada). As canaletas formadas pelos círculos em alto relevo ajudam na separação de glomerosporos facilitando sua contagem. Embora haja estes auxílios é comum que haja erros e frequentemente uma segunda contagem na mesma amostra resulta em valor diferente. Levando-se em consideração o número de amostras que normalmente são analisadas, a contagem destas estruturas diminutas trata-se de um trabalho cansativo e desgastante.

\section{SOLUÇÃO DE PROBLEMA}

\section{III.1. PREPARAÇÃO DO AMBIENTE.}

Para desenvolvimento deste trabalho foi utilizado o software, Scilab 5.4.1 versão 64 bits pré-compilada baixada a partir do site www.scilab.org. Também foi utilizado um módulo adicional o SIVP - Scilab Image and Video Processing Toolbox este módulo possui funções para o processamento de imagem e foi utilizado para facilitar leitura e gravação das imagens. Este módulo pode ser instalado a partir do próprio Scilab com seu gerenciador de módulos ATOMS.

Figura 1: Imagem (430x311) esporos fungo Claroidwh

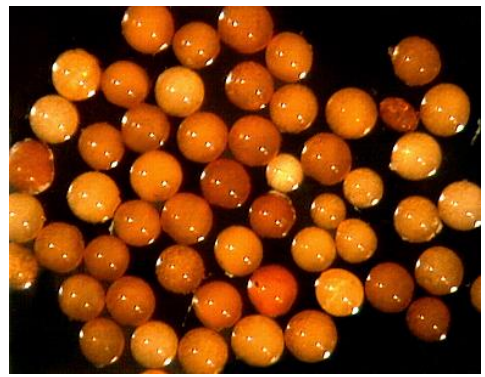

Fonte: www.invam.wvu.edu

ReCiC- Revista de Ciência da Computação
Figura 2: Imagem (387x303) esporos fungo Laeviswh

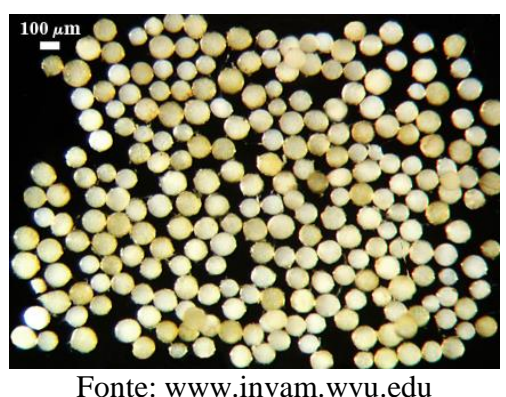

\section{III.2. AS IMAGENS}

Para desenvolvimento do trabalho foram utilizadas imagens do INVAM (International Culture Collection of Arbuscular Mycorrhizal Fungi) pertencente a Universidade Oeste da Virginia (WVU). As imagens foram retiradas do Site: invam.wvu.edu.

As imagens dos esporos apresentam algumas características similares. Todas as imagens têm aproximadamente $400 \times 300$ pixel de tamanho e um fundo escuro tendendo ao preto. Os esporos estão dispostos na imagem de forma que muitos estão próximos entre si chegando a se tocarem e em alguns casos sobrepondo parte um do outro. A cor dos esporos e o tamanho não apresentam características muito similares, dentro do conjunto de imagens são encontradas várias cores e tamanhos. As figuras, Figura 1 e Figura 2 mostram estas características.

\section{III.3. DESENVOLVIMETO DOS ALGORITMOS}

A maioria dos códigos desenvolvidos tomou como base as definições dos operadores fuzzy ANDRADE (2014) e das funções de normalização. Outros algoritmos foram desenvolvidos como a máscara de corte, conversão da imagem para binário, função de contagem e funções que auxiliaram o processo como um todo. A seguir descreveremos alguns dos algoritmos.

Dois algoritmos foram desenvolvidos para transformar o espaço de valores da imagem. A função de normalização converte os valores dos pixels que estão no intervalo [0, 255] para o intervalo real $[0,1]$ possibilitando que a imagem seja processada por operadores morfológicos e a função de "desnormalização" retorna os valores para o intervalo $[0,255]$ para que a imagem possa ser remontada. As funções matemáticas usadas foram:

$$
\begin{gathered}
F(x)=x / 255 \\
F(x)=\sin x \\
F(x)=\tan x
\end{gathered}
$$

\section{III.3.1 OPERADORES}

Os algoritmos dos operadores seguem uma estrutura básica igual para todos mudando apenas o seu núcleo onde se calcula o valor do pixel o que em cada caso é definido para cada implicação de Goguen, Gödel e Lukasiewicz.

O Trecho código 1, Figura 3 mostra a estrutura básica do código dos operadores, este trecho contém dois laços 'for' necessários para percorrer os pixels da imagem e outros 
dois laços 'for' para percorrer os pixels do elemento estruturante. Dentro do laço mais interno o valor do pixel é calculado e posteriormente o valor é armazenado na matriz resultante.

Figura 3: Trecho código 1. Algoritmo que percorre a imagem e o elemento.

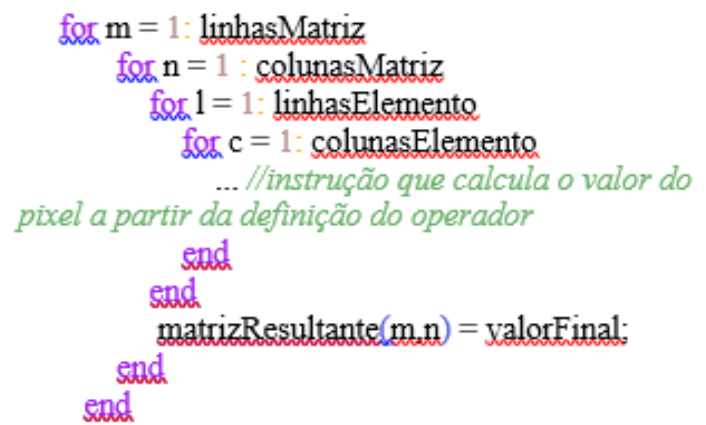

Fonte: o autor

O algoritmo inicialmente tem ordem de complexidade $n^{4}$ considerando que a imagem e o elemento tem tamanho desconhecido, no entanto, para contagem de esporos de fungos o elemento estruturante vai ser definido de forma que seu tamanho e formato serão conhecidos o que leva a ordem de complexidade do algoritmo a ser reduzida para $n^{2}$. Os algoritmos que calculam o valor do pixel foram codificados a partir das definições os operados e suas implicações.

Figura 4: Trecho código 2. Algoritmos que implementam as definições dos operadores Lukasiewicz.

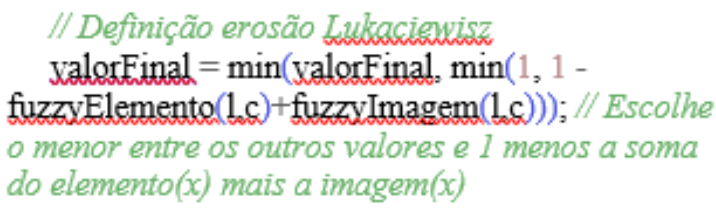

\section{// Definição erosão Lukaciewisz}

valorFinal $=\max ($ valorFinal $\max (0$, fuzzyElemento $\left(1_{c} \mathrm{c}\right)+$ fuzzuImagem $\left.(1 \mathrm{c})-1\right)$ ); //

Escolhe o maior entre os outros valores e a soma do elemento $(x)$ mais a imagem $(x)$ menos 1

\section{Fonte: o autor}

O trecho de código 2, Figura 4 mostra a codificação das definiçõos de dilatação e erosão para a implicação de Lukasiewicz usada neste trabalho.

\section{III.3.2 MÁSCARA DE CORTE}

O algoritmo máscara de corte foi desenvolvido com a finalidade de remover pequenos pontos e linhas estreitas dentro de imagens binárias. Seu conceito foi baseado nas definições de vizinhança $N_{8}(p)$.

A máscara funciona analisando a adjacência de cada pixel para decidir se ele pertence a uma grande estrutura ou se ele é apenas um pequeno ponto ou traço estreito. A análise é realizada verificando-se os pixels nos extremos das retas diagonais, vertical e horizontal a uma determinada distância de um pixel central. Caso em uma destas retas o pixel central seja preto e os pixels nos extremos sejam brancos o pixel central é convertido para branco. A Figura 5 ilustra os pontos de análise do algoritmo para uma distância 1.

Figura 5: (a) Análise diagonal 1, (b) Análise diagonal 2, (c) Analise diagonal 3 (d) Analise diagonal 4.

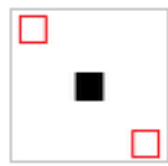

(a)

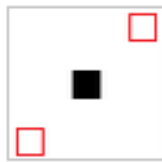

(b)

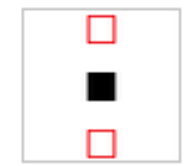

(c)

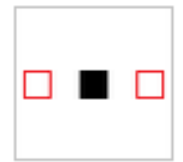

(d)
Fonte: o autor

\section{III.3.4. CÓDIGOS DE CONTAGEM DE OBJETOS}

A função de contagem de objetos tem como objetivo contar a quantidade de objetos e calcular seu tamanho em pixel (quantidade de pixel do objeto) dentro de uma imagem binária.

O algoritmo foi implementado seguindo as definições de vizinhança $N_{4}(p)$ onde apenas os vizinhos horizontais e verticais são considerados. $\mathrm{O}$ algoritmo percorre toda a matriz linha por linha e quando um pixel preto é encontrado ele recebe um número que será seu identificador como objeto e os pixels vizinhos a estes também receberam o mesmo número. A Figura 6 exemplifica essa marcação do algoritmo.

Figura 6: Imagem representa a marcação do algoritmo de contagem.

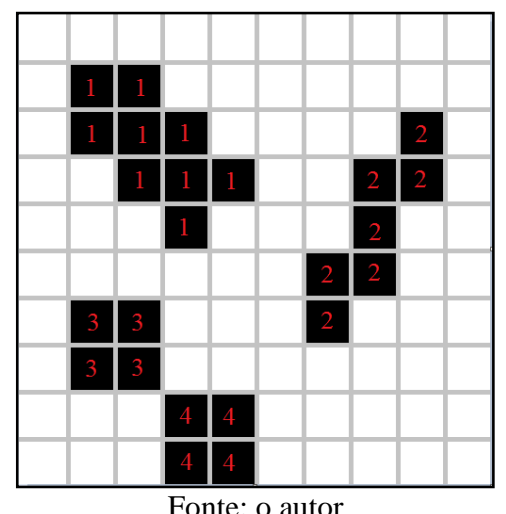

Após a marcação, a quantidade de objetos será o número de identificação do último objeto marcado e o tamanho de cada objeto é calculado somando os pixels marcados com mesmo identificador. Estes dois valores são importantes para se estimar o tamanho médio dos esporos.

\section{III.4. PROCESSO}

O processo de quantificação de esporos de fungo por meio de imagens digitais se divide em duas partes: a primeira visa separar os esporos na imagem por meio de operadores 
morfológicos, transformação para imagem binária e uso de máscara; a segunda consiste em contar esporos separados dentro da imagem. A Figura 8, mostra todas as etapas do processo de quantificação desde a separação até a imagem usada para contagem.

Figura 7: (a) Imagem original, (b) Imagem processada pelos operadores, (c) Imagem convertida para binário, (d) Imagem processada pela máscara de corte.

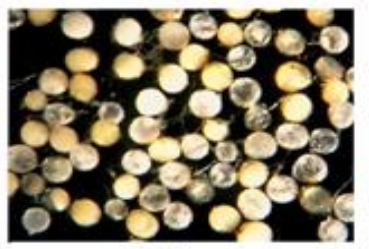

(a)

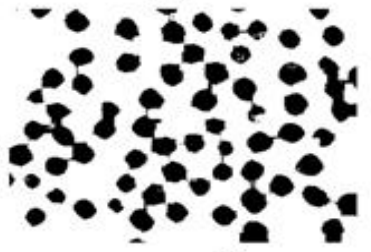

(c)

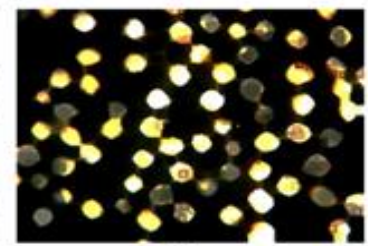

(b)

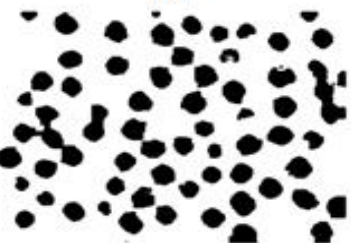

(d)
Fonte: o autor

A imagem original (a) é processada por meio de operadores morfológicos a fim de separar os esporos que estejam juntos, em seguida, a imagem resultante (b) é convertida para binária (c) na qual o fundo se torna branco e os possíveis esporos ficam pretos, novamente a imagem é processada para retirada de finas linhas que ainda liguem esporos vizinhos e pequenos pontos que tenham surgido durante o processo. A imagem resultante (d) de todo esse processo é a imagem que passa pelo processo de contagem.

\section{III.4.1 PROCESSO DE SEPARAÇÃO}

O processo de separação consiste no uso de operadores morfológicos fuzzy e outros algoritmos auxiliares que têm como finalidade afastar objetos próximos dentro da imagem. As três variáveis principais do processo são: o operador morfológico, a morfologia e o elemento estruturante. A seguir são mostrados os passos tomados para definir estas variáveis.

\subsubsection{TESTES DOS OPERADORES}

Para a escolha do operador foi montada uma tabela com todas as possibilidades de operações possíveis (18 combinações) tais possibilidades são mostradas na Tabela 1. O operador morfológico de erosão tem como característica diminuir a imagem e o operador de dilatação aumentar, desta forma, podemos utilizar a erosão para separar os esporos e a dilatação para uma possível reconstrução de esporos pequenos. Para visualizar como estas características se comportam nas imagens dos esporos uma sequência de até três processamentos seguidos foram realizados em uma imagem com todas as combinações de modos de operadores da Tabela 1 o que resultou em 6.175 imagens. A Figura 8 mostra alguns dos resultados obtidos com o processo.

Tabela 1: Tabela de operações possíveis

\begin{tabular}{cccc}
\hline $\mathbf{N}$ & Operador & Morfologia & $\begin{array}{c}\text { Função para } \\
\text { normalizar }\end{array}$ \\
\hline $\mathbf{1}$ & Erosão & Gödel & $\mathrm{x} / 255$ \\
$\mathbf{2}$ & Erosão & Gödel & seno(x) \\
$\mathbf{3}$ & Erosão & Gödel & tangente(x) \\
$\mathbf{4}$ & Erosão & Goguen & $\mathrm{x} / 255$ \\
$\mathbf{5}$ & Erosão & Goguen & seno(x) \\
$\mathbf{6}$ & Erosão & Goguen & tangente(x) \\
$\mathbf{7}$ & Erosão & Lukasiewicz & $\mathrm{x} / 255$ \\
$\mathbf{8}$ & Erosão & Lukasiewicz & seno(x) \\
$\mathbf{9}$ & Erosão & Lukasiewicz & tangente(x) \\
$\mathbf{1 0}$ & Dilatação & Gödel & $\mathrm{x} / 255$ \\
$\mathbf{1 1}$ & Dilatação & Gödel & seno(x) \\
$\mathbf{1 2}$ & Dilatação & Gödel & tangente(x) \\
$\mathbf{1 3}$ & Dilatação & Goguen & $\mathrm{x} / 255$ \\
$\mathbf{1 4}$ & Dilatação & Goguen & seno(x) \\
$\mathbf{1 5}$ & Dilatação & Goguen & tangente(x) \\
$\mathbf{1 6}$ & Dilatação & Lukasiewicz & $\mathrm{x} / 255$ \\
$\mathbf{1 7}$ & Dilatação & Lukasiewicz & seno(x) \\
$\mathbf{1 8}$ & Dilatação & Lukasiewicz & tangente(x) \\
\hline & & Fonte: o autor &
\end{tabular}

Nas imagens resultantes foi possível observar que os operadores se comportam como o esperado onde o operador de erosão diminuiu os esporos e o de dilatação os aumentou. Desta forma, fica claro que o uso do operador de erosão é ideal para a separação dos esporos, no entanto, a eficácia do operador de dilatação para reconstrução de pequenos esporos fica reduzida já que ao mesmo tempo em que aumenta estes esporos acaba juntando novamente esporos separados.

Figura8: (a) Esporos fungo Claroidwh, (b) Elemento estruturante, (c) Resultado operação modo 1, (d) Resultado operação em sequência modo 4-2, (e) Resultado operação em sequência modo 4-16-18.
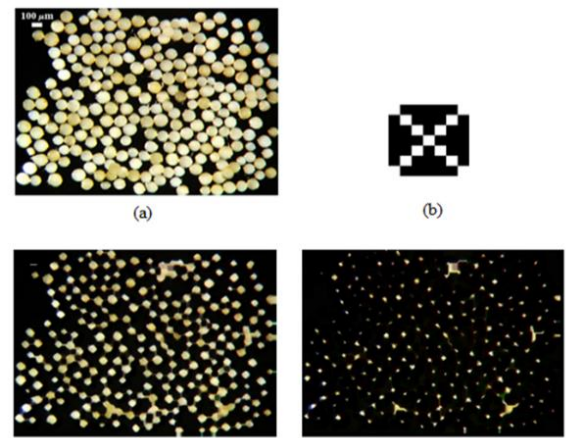

(c)

(d)

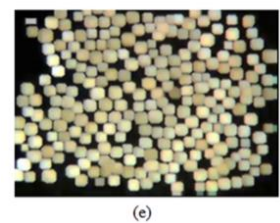

Fonte: o autor 


\section{III.4.3 ESCOLHA DA MORFOLOGIA}

Com a confirmação das características dos operadores nas imagens de fungos o passo seguinte deve ser determinar quais das morfologias separam melhor. Para escolha da melhor morfologia, novos testes foram realizados em 37 imagens retiradas do site: invam.caf.wvu.edu, nestes testes são levados em conta o quanto o processo separa os fungos e qual a porcentagem de acerto na contagem. Cinco testes foram realizados utilizando os operadores $\mathrm{e}^{x} / 255$ como função de normalização. A contagem foi realizada utilizando o método de contagem de objetos em imagem binária e a aproximação pela média. Estes métodos serão explicados mais à frente.

Os 5 testes foram realizados da seguinte forma:

- Teste 1- sequência de cinco erosões e uma dilatação utilizando um elemento de 3x3pixel;

- Teste 2- sequência de quatro erosões e uma dilatação utilizando um elemento de 3x3pixel;

- Teste 3- sequência de duas erosões e uma dilatação utilizando um elemento de 7x7pixel;

- Teste 4- sequência de quatro erosões e uma dilatação utilizando um elemento de $5 \times 5$ pixel;

- Teste 5- sequência de três erosões e uma dilatação utilizando um elemento de 7x7pixel.

A Figura 9 mostra os elementos usados nos testes.

Figura 9: (a) Elemento usado no teste 1, (b) Elemento usado no teste 2, (c) Elemento usado no teste 3, (d) Elemento usado no teste 4, (e) Elemento usado no teste 5 .

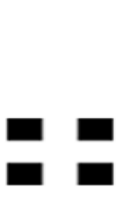

(a)

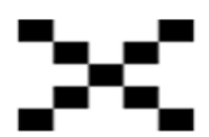

(d)

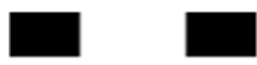

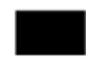

(c)

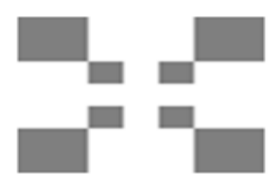

(e)
Fonte: o autor

A partir dos testes foi possível concluir que a morfologia de Gödel separou melhor os esporos e, assim, obteve-se melhor resultados na contagem.

Analisando a quantidade de operações e elemento estruturante, pode se fazer uma relação em que a quantidade de operações é inversamente proporcional ao tamanho do elemento, pois nos casos em que o elemento usado foi maior e a quantidade de operações foi menor o resultado ainda se manteve próximo dos demais. Já a mudança na cor do elemento mostra que quanto mais clara

ReCiC- Revista de Ciência da Computação a cor, maior o número de operações necessárias para separar os fungos de forma satisfatória. A Tabela 2 mostra quantas das imagens tiveram acerto acima de $80 \%$ para cada teste realizado.

Tabela 2: Resultados dos testes implicação/conjunção

\begin{tabular}{cccc}
\hline \multicolumn{4}{c}{$\begin{array}{c}\text { Número de imagens com contagem acima de } 80 \% \text { de } \\
\text { acerto sobre um conjunto de } 37 \text { imagens }\end{array}$} \\
\hline Teste & $\begin{array}{c}\text { Implicação } \\
\text { Conjunção }\end{array}$ & $\begin{array}{c}\text { Contagem } \\
\text { simples }\end{array}$ & $\begin{array}{c}\text { Contagem } \\
\text { media }\end{array}$ \\
$\mathbf{1}$ & Gödel & 23 & 28 \\
$\mathbf{1}$ & Goguen & 10 & 21 \\
$\mathbf{1}$ & Lukasiewicz & 13 & 8 \\
$\mathbf{2}$ & Gödel & 14 & 24 \\
$\mathbf{2}$ & Goguen & 5 & 18 \\
$\mathbf{2}$ & Lukasiewicz & 13 & 10 \\
$\mathbf{3}$ & Gödel & 12 & 26 \\
$\mathbf{3}$ & Goguen & 12 & 26 \\
$\mathbf{3}$ & Lukasiewicz & 13 & 24 \\
$\mathbf{4}$ & Gödel & 22 & 25 \\
$\mathbf{4}$ & Goguen & 24 & 23 \\
$\mathbf{4}$ & Lukasiewicz & 23 & 15 \\
$\mathbf{5}$ & Gödel & 11 & 9 \\
$\mathbf{5}$ & Goguen & 16 & 17 \\
$\mathbf{5}$ & Lukasiewicz & 2 & 2 \\
\hline & & Fonte: o autor & \\
\hline
\end{tabular}

\section{III.4.4 ESCOLHA DO ELEMENTO E USO DA MÁSCARA DE CORTE DE LINHA.}

Durante os testes para escolha da morfologia o elemento estruturante mostrou-se de vital importância para o processo de separação e agora focaremos em sua escolha. A partir deste ponto a máscara de corte de linhas foi implementada e incluída no processo e assim os fungos que ficam juntos por linhas depois do processamento por operadores e conversão para binária podem ser separados por esta máscara.

Para escolha do parâmetro de distância utilizado na máscara, as imagens binárias resultantes do processo de escolha da morfologia foram processadas com parâmetros de distância de 1 a 4 . A partir das análises visuais dos resultados, o parâmetro de distância 2 mostrou-se mais eficiente apagando a maioria das linhas e não apagando muitos fungos que no processo de separação ficaram muito pequenos.

Sobre a escolha do elemento sabemos que aumentando o tamanho do elemento o número de erosões pode ser diminuído então os testes foram feitos com apenas uma erosão o que reduziu de 2 a 4 vezes o tempo de execução para o processo de contagem. A dilatação foi removida do processo, pois este operador era usado para recuperar a forma característica dos fungos após as erosões e, no entanto, não se mostrou eficaz para a contagem tendo em vista que ele voltava a juntar os fungos.

Nove elementos de tamanho entre 9x9 e 15x15 pixels e cores variando dentro da escala de cinza foram usados. A 
Tabela 3 mostra o resultado dos testes e a Figura 10 mostra os elementos usados.

Tabela 3: Resultados dos testes com os elementos

\begin{tabular}{lllll}
\hline $\begin{array}{l}\text { Número de imagens com contagem acima de } 80 \% \\
\text { de acerto sobre um conjunto de } 37 \text { imagens }\end{array}$ \\
\hline Elemento & $\begin{array}{l}\text { Cont } \\
\text { simpl } \\
\text { es }\end{array}$ & $\begin{array}{l}\text { Cont } \\
\text { medi } \\
\text { a }\end{array}$ & $\begin{array}{l}\text { Cont. } \\
\text { pós } \\
\text { masc } \\
\text { ara }\end{array}$ & $\begin{array}{l}\text { Cont. } \\
\text { media } \\
\text { pós } \\
\text { mascar } \\
\text { a }\end{array}$ \\
& & & 22 & 22 \\
(a) & 18 & 19 & 23 & 23 \\
(b) & 16 & 19 & 21 \\
(c) & 13 & 12 & 19 & 21 \\
(d) & 6 & 6 & 4 & 8 \\
(e) & 10 & 9 & 11 & 15 \\
(f) & 12 & 13 & 18 & 20 \\
(g) & 14 & 15 & 17 & 21 \\
(h) & 7 & 10 & 17 & 19 \\
(i) & 6 & 8 & 18 & 19 \\
\hline \multicolumn{5}{c}{ Fonte: o autor } \\
\hline
\end{tabular}

Figura 10: $(\mathrm{a}, \ldots, \mathrm{i})$ elementos usados nos testes para escolha de elemento.

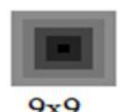

(a)

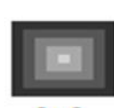

$9 \times 9$

(f)

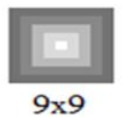

(b)

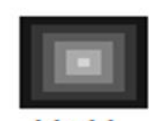

$1 \times 1$
$(g)$

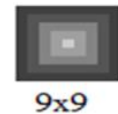

(c)

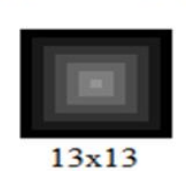

(h)

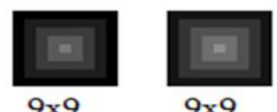

(e)
Fonte: o autor

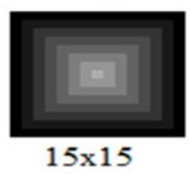

(i)

O uso de elementos mais escuros ou muito grandes acabou se mostrando pouco eficaz para separar os fungos, tais elementos acabaram apagando os fungos na imagem ou tornando-os muito pequenos, o que impossibilitou a sua contagem, pois a máscara pode apagá-los. A Figura 11 mostra a diferença de resultado entre dois elementos. $\mathrm{O}$ elemento (b) pequeno e claro e o elemento (i) grande e escuro.

Figura 11: (1) Imagem original, (2) Resultado com elemento (b), Resultado com elemento (i).

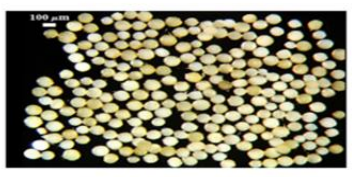

(1)

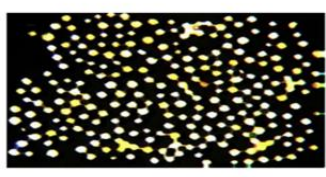

(2)

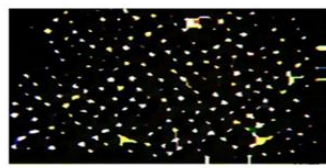

(3)

Fonte: o autor

Ao fim deste processo o elemento (b) foi escolhido como melhor opção para a contagem e a imagem do elemento foi transformada em uma matriz e acrescentada ao código como uma constante do processo.

\section{III.4.5 PROCESSO DE SEPARAÇÃO FINALIZADO}

Com a escolha do operador, da morfologia e do elemento o processo de separação pode ser concluído com a inclusão da conversão para binário e da máscara de corte ficando o processo configurado da seguinte maneira:

1- utilizar erosão Gödel com elemento(b).

2- converter para binário.

3- utilizar máscara de corte com parâmetro de distância 2.

\section{III.5 PROCESSO DE CONTAGEM}

O processo de contagem consiste em contar objetos em uma imagem binária. No caso deste trabalho os objetos serão os esporos.

\subsubsection{PROCESSO DE CONTAGEM SIMPLES}

O processo de contagem simples faz apenas a contagem dos objetos na imagem utilizando o algoritmo de contagem sem dar importância ao tamanho de cada objeto, neste caso um objeto de 1 pixel de tamanho e outro com 100 pixels de tamanho tem o mesmo peso na contagem. A Figura 12 mostra o problema deste tipo de contagem onde o objeto circulado em vermelho tem o mesmo peso dos demais, no entanto, o objeto maior corresponde a 4 esporos que não foi possível separar no processo.

Figura 13: Fragmento de imagem de esporos de fungos Claroidwh após o processo de separação.

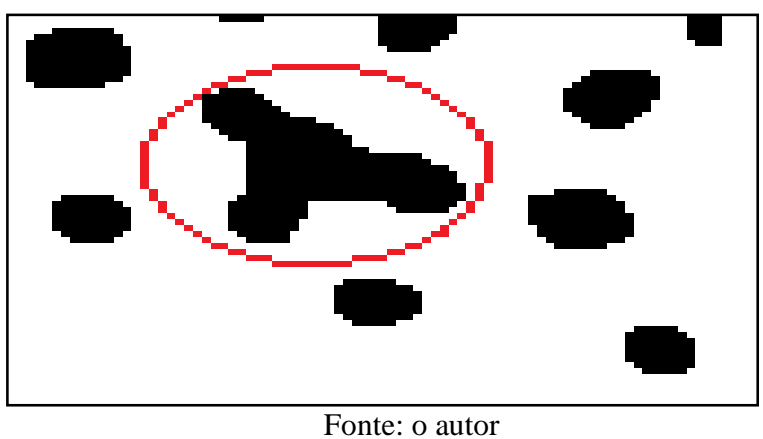

\section{III.5.2 PROCESSO DE CONTAGEM POR MÉDIA}

No processo de contagem simples ao não levar em conta o tamanho do objeto, esporos que ficaram juntos ou pedaços pequenos de algum esporo que se separou são contados de forma errônea por esse motivo uma verificação desses objetos se faz necessária.

Para descobrir se um objeto representa um único, vários ou um pequeno pedaço de esporo foi feito um cálculo médio do tamanho destes objetos utilizando a quantidade de pixels que cada um tem. Considerando que vários esporos únicos foram separados o cálculo médio terá valor próximo ao tamanho do esporo.

ReCiC- Revista de Ciência da Computação 
A partir da media $\mathrm{M}$ e de cada conjunto $\mathrm{Q}$, a quantidade de esporos $\mathrm{P}$ é calculada da seguinte forma:

1. calcula-se a média $\mathrm{M}$ do tamanho dos objetos (esporos)

$M=$ Quantidade de pixels pretos / quantidade de objetos.

2. calcula-se os esporos $P_{1}$ inteiros descartando o resto da divisão.

$P_{1}={ }^{(Q)} /{ }_{M}$ (divisão inteira sendo R o resto)

3. calcula-se o resto $\mathrm{R}$ da divisão.

$R=(\mathrm{Q}$ Modulo $\mathrm{M})($ resto da divisão $)$

4. calcula-se, se o resto corresponde a um esporo menor que a média.

$P_{2}=(R+(M * 7 / 100) / M)$

(soma aqueles que são superiores a 93\% da media )

Por último soma-se os dois valores.

$P=P_{1}+P_{2}$;

Com este recurso a contagem obteve melhores resultado, o que pode ser observado nas tabelas, Tabela 2 e Tabela 3.

\section{CONCLUSÃO}

O processo de separação se mostrou satisfatório na maioria das fotos utilizadas nos testes, entretanto, em algumas delas a separação foi ineficiente. A falta de um padrão de cores e de tamanho dos esporos foi o fator mais relevante que afetou negativamente o processo de separação uma vez que um mesmo elemento estruturante traz resultados diferentes para padrões diferentes. Uma alternativa para aumentar a eficiência do processo de separação seria a escolha de um elemento estruturante para cada tipo de fungo.

No que tange o processo de contagem, a contagem simples não se mostra muito eficiente tendo em vista que conta objetos de tamanhos muito diferentes atribuindo-lhes o mesmo valor, já a contagem por média é uma melhoria da outra e ao utilizar a média do tamanho dos objetos (esporos) para calcular o valor de cada na contagem traz uma significativa melhora. Esse método, no entanto, é bastante influenciado pela separação, pois para que a média se aproxime do valor real, uma boa quantidade de esporos deve estar separada..

Como resultados das implementações, um toolbox para a contagem foi desenvolvido e seu código acompanha este trabalho.

Este artigo apresentou um método de quantificação de esporos de fungos micorrízicos, utilizando morfologia matemática fuzzy para separação dos esporos e algoritmos para contagem. Verificou-se que os operadores morfológicos fuzzy se mostraram eficientes para o processo de separação dos fungos e que o processo de contagem automática pode ser uma forma alternativa à contagem manual tendo em vista que o processo alcançou acertos acima de $80 \%$.

Todos os algoritmos foram desenvolvidos na ferramenta Scilab que é um software livre de código aberto o que pode facilitar a utilização do método por outros desenvolvedores e pesquisadores.

\section{REFERÊNCIAS}

[1] ANDRADE, A.O. Um Sistema de Contagem baseado em Morfologia Matemática Fuzzy. Tese de doutorado (Ciências) Universidade Federal Do Rio Grande Do Norte, Natal. 2014.X2. Author, Title of the Book, Publishing House, 200X.

[2] ANDRADE, A.O., R.M.P. TRINDADE, D.S.MAIA, R.H.N.SANTIAGO \& A. M. G. GUEREIRO (2012), Uso da morfologia matemática fuzzy na contagem esporos de fungos micorrízicos, em 'Recentes Avanços em Sistemas Fuzzy', II Congresso Brasileiro de Sistema Fuzzy.

[3] BOUCHET A., PASTORE J. e BALLARIN V. Segmentation of Medical Images using Fuzzy Mathematical Morphology. JCS T Vol. 7 No. 3. 2007. Disponível em: $<$ http://www.fmi.uni-

sofia.bg/courses/biomed/morphgeom/papers/JCST.pdf>. Acesso em: 15 ago. 2014.

[4] BARBEDO, J.G.A. (2012), Estado da Arte das Técnicas de Contagem de Elementos Específicos em Imagens Digitais, Embrapa.

[5] SALIS, T.T. \& G.A.S. PEREIRA. (2006), Contagem automática de tarugos de aço por meio de visão computacional., Dissertação de mestrado, Universidade Federal de Minas Gerais. Disponível em: <http://www.ppgee.ufmg.br/documentos/PublicacoesDefesas/ 786/ContagemAutomatica.pdf > . Acesso em: 25 ago. 2014

[6] POOMCOKRAK, J. \& C. NEATPISARNVANIT (2008), 'Red blood cells extraction and counting.',International ymposium on Biomedical Engineering, 3(199-203). Porter, W.M. (1979), 'The"most

[7] MEN, H.; WU, Y.; LI, X.; KOU, Z.; YANG, S. Counting method of heterotrophicbacteria based on image processing. In: IEEE Conference on Cybernetics and Intelligent Systems, 7., 2008, Chengdu. Proceedings... London: IEEE: Piscataway, 2008. p. 1238-1241.

[8] BERBARA, R.L.L. \& FONSECA, H.M.A.C. Colonizacão e esporulacão de fungos micorrízicos arbusculares in vitro. In: SIQUEIRA, J.O., ed. Avanços em fundamentos e aplicação de micorrizas. Lavras, Universidade Federal de Lavras, 1996. p.39-65.

[9] GOTO B.T e MAIA L.C.. Glomerospores: A new denominatio for the spores of glomeromycota, a group molecularly distinc from the zygomycota. Mycotaxon, (96):129-132, 2006. Disponível em:

file:///C:/Users/alecio/Downloads/Goto\%20et\%20a1\%202013 _Acaulospora_endographis\%20(1).pdf> . Acessado em: 18 ago. 2014.

[10] REDECKER D., SCHUBLER A., STOCKINGER H., STUMER S.L., MORTON J.B., and WALKER C. An evidence-based consensus for the classification of arbucular mycorrhizal fungi (glomeromycota). Mycorrhiza, 2013. Disponível

em:< http://books.google.com.br/books?id=2LPrAgAAQBAJ\&pg= $\mathrm{PA} 288 \& \operatorname{lpg}=\mathrm{PA} 288 \& \mathrm{dq}=$ An+evidence -

ased+consensus+for+the+classification+of+arbuscular+myco rrhizal+fungi\&source=bl\&ots $=\mathrm{aFqvi}-\mathrm{ooJH} \&$ sig $=-$ GYDX7JdzTdA-fybhCeJJPo6KqM\&hl=pt-

BR\&sa $=X \&$ ei $=n V V 3 V O i J K Y e j N s f n g-$

AC\&ved=0CHUQ6AEwCQ\#v=onepage $\& \mathrm{q}=\mathrm{An} \% 20$ evidence based $\% 20$ consensus $\% 20$ for $\% 20$ the $\% 20$ classification $\% 20$ of $\%$ 20 arbuscular $\% 20$ mycorrhizal $\% 20$ fungi $\& \mathrm{f}=$ false $>$. Acessado em: 18 ago. 2014.

[11] SOUZA, F.A., STUMER, S.L., CARRENHO R., and TRUFEM,S.F.B. Micorrizas: 30 anos de pesquisas no Brasil, chapter Classificação e taxonomia de fungos micorrízicos arbusculares e sua diversidade e ocorrência no Brasil, pages 1573. Editora UFLa, 2010. 